\title{
Contrast sensitivity before and after small incision lenticule extraction and femtosecond laser in situ keratomileusis
}

\author{
Asik Pradhan', Kishore Raj Pradhan', Gauri Shankar Shrestha², \\ Purushottam Dhunganga', Raju Kaiti ${ }^{3}$ \\ ${ }^{1}$ Refractive Surgery Unit, Tilganga Institute of Ophthalmology, Gaushala, \\ Kathmandu, Nepal; ${ }^{2}$ Optometry Unit, Nepal Eye Hospital, Kathmandu, Nepal; \\ ${ }^{3}$ Department of Ophthalmology, Institute of Medicine, Maharajgunj Medical \\ Campus, Tribhuvan University, Kathmandu Nepal.
}

\begin{abstract}
Introduction: Contrast sensitivity function after laser ablation of the cornea in refractive surgery is an important tool for measuring quality of visual function. The effect of small incision lenticule extraction (SMILE) and femtosecond laser in situ keratomileusis (FS-LASIK) on visual function can be compared by measuring spatial contrast sensitivity.
\end{abstract}

Purpose: This study was to compare contrast sensitivity function in patients undergoing refractive surgery for myopia at Tilganga Institute of Ophthalmology.

Methods: In a retrospective study, 15 subjects who underwent refractive surgeries comprising 9 cases of SMILE and 6 cases of FS-LASIK at Tilganga Eye Hospital were enrolled in the study. A major assessment included best corrected monocular contrast sensitivity with functional vision analyzer at spatial frequencies of 1.5, 3, 6, 12, and $18 \mathrm{cycles} / \mathrm{deg}$ in photopic condition $(85 \mathrm{~cd} / \mathrm{m} 2)$ before, 3 months, and 1 year after the surgery. The average functional acuity contrast test scores for each spatial frequency were recorded. Differences between preoperative and postoperative contrast sensitivity at each spatial frequency were analyzed through parametric paired Student's t-test.

Results: Contrast sensitivity of postoperative FS-LASIK and SMILE did not differ from preoperative values at a photopic level. However, at high spatial frequency (12 and 18 cycles/deg), contrast sensitivity function improved significantly $(p<0.05)$ in eyes after FS-LASIK. Eyes after SMILE surgery did not show any reduction in contrast sensitivity at all the spatial frequencies.

Conclusion: Under photopic conditions, the contrast sensitivity function is unaffected by SMILE or FS-LASIK.

Keywords: contrast sensitivity, FS-LASIK, myopia, SMILE

Correspondence: Gauri Shankar Shrestha, M.Optom, FIACLE, Assistant Professor of Optometry, Department of Ophthalmology, B.P. Koirala Lions Center for Ophthalmology Institute of Medicine, Tribhuvan University, Kathmandu, Nepal. E-mail: gs101lg@hotmail.com 


\section{Introduction}

Excimer laser refractive surgery aims to reproduce the preoperative optical performance while making the eye emmetropic. Though technology and refractive surgeons have made great strides toward establishing emmetropia postoperatively, they have not been able to duplicate the optical performance previous to surgery. Some studies have reported visual problems such as haze, halos, stray light images, or glare in patients following refractive surgery, ${ }^{1-9}$ complications that cause significant decreases in visual performance. Efforts have been made to achieve better results by modifying some characteristics of the surgery such as transition zones, multistep ablations, and new techniques like femtosecond laser in situ keratomileusis (FS-LASIK) and small incision lenticule extraction (SMILE).

LASIK has evolved from a variety of techniques in refractive surgery. LASIK combines a technique of creating a hinged corneal flap from the automated lamellar keratectomy with excimer laser ablation from the photorefractive keratectomy (PRK). In early days, a microkeratome was used to create corneal flaps. But, nowadays, it has been replaced by femtosecond laser that creates precise and desired corneal flaps. Since Food and Drug Administration approval of an ultrafast laser in 2000, the femtosecond laser has transfigured the creation of flaps for LASIK. ${ }^{10-13}$ The pulse duration of the femtosecond laser is in the $10^{-15}$ second range. The 1,053 nm wavelength of light used by the laser is not absorbed by optically transparent tissues. Moreover, it can be focused anywhere within the cornea where the energy can be raised to a threshold such that a plasma is generated. ${ }^{14}$ The corneal flap is kept aside and the excimer laser is applied to reshape the surface of the cornea by removing anterior stromal tissue. The word excimer is an abbreviation of the term "excited dimer." The excimer laser produces a beam of ultraviolet energy at various wavelengths depending upon the gas elements used. The $193 \mathrm{~nm}$ ultraviolet light from the argon fluoride laser, which has the least corneal transmission, causes less adjacent tissue damage and creates a smoother ablation than longer wavelength lasers. ${ }^{15}$ At a wavelength of $193 \mathrm{~nm}$, high-energy photons break organic molecular bonds of the superficial corneal tissue in a process called ablative photodecomposition. ${ }^{16,17}$ Further improvement in lasers occurs with eye-tracking systems that allow precise corneal ablation during eye movement. ${ }^{18}$

SMILE involves the use of a femtosecond laser to create a corneal lenticule that is extracted whole through a small incision without the use of an excimer laser. In 2007, an intrastromal lenticule method was reintroduced as an alternative to LASIK called femtosecond lenticule extraction (FLEx) and was intended for patients with extreme myopia. After improvements to scan modes and energy parameters, improved visual recovery times were noted (stability and predictability of the refractive outcome, improvement in visual acuity with lesser 
complications), with refractive results similar to LASIK. ${ }^{19}$ Following the implementation of FLEx, a procedure called SMILE was developed, which involves passing a dissector thorough a small 2 to $3 \mathrm{~mm}$ incision to separate the lenticular interfaces and allow extraction of the lenticule without a need to create a flap. ${ }^{20}$

Contrast sensitivity is a very important measure of visual function, especially in situations of low light, fog, or glare, when the contrast between objects and their background often is reduced. It provides us information about the visibility of objects that vary in size, contrast, and orientation. Loss of contrast sensitivity can occur at high, low, and broad spatial frequencies. Various ocular and systemic diseases can affect contrast sensitivity functions in different ways and at different frequencies. Changes in contrast sensitivity can also be observed in the refractive surgeries that could be related to an increase in higher-order aberrations such as coma and trefoil. When there is a loss of contrast sensitivity, light entering the eye does not focus uniformly on to the retina. Instead, some of the light scatters and the vision in that eye can appear washed out and/or foggy. Loss of contrast sensitivity can range from not noticeable to extremely annoying. ${ }^{21}$

Montés-Micó et al. ${ }^{22-25}$ reported that visual function after refractive surgery can be documented by measuring spatial contrast sensitivity. It assesses the combined visual impact of any light scattering, optical aberration, or defocus that may occur following refractive surgery. ${ }^{6,8-9,26}$ However, in order to explore the visual performance in patients before and after refractive surgery, mesopic contrast sensitivity function should be evaluated. ${ }^{26}$ These authors found significant reduction in contrast sensitivity under mesopic conditions following PRK and LASIK, even though the photopic contrast sensitivity function was normal.

FS-LASIK and SMILE are now available in Nepal. Though FS-LASIK and SMILE provide normal uncorrected visual acuity, the visual performance in different illuminations is largely unknown in our context. As the contrast sensitivity function is one of the major determinants of visual performance, the present study was conducted to compare contrast sensitivity function among patients before and after SMILE and FS-LASIK.

\section{Materials and methods}

This study was a retrospective, longitudinal, and comparative analysis of 15 subjects who underwent SMILE (nine subjects) and FS-LASIK (six subjects) for correction of myopia and myopic astigmatism at Tilganga Institute of Ophthalmology, Kathmandu, in July and August 2014. All subjects were programmed for SMILE and LASIK procedure. The procedure that gave the maximum correction with minimum amount of tissue loss was selected as an operating procedure for that individual subject. The mean age of subjects undergoing FS-LASIK was 22.0 (SD 3.8) years, with the age ranging between 18 and 27 years. The mean age of 
subjects selected for SMILE was 24.8 (SD 4.6) years, with the age ranging between 19 and 30 years.

All the subjects who were enrolled in the study had stable refraction, keratometry, and pachymetry at least for 12 months and did not have dry eye, any preoperative medication other than those prescribed, active diseases of ocular surface, and adnexa. No patient had ocular morbidities that could affect contrast sensitivity such as glaucoma, corneal and neuro-ophthalmic diseases, or cataracts. Those patients who were lost to follow-up during 3 and 12 months were excluded. All patients received a detailed explanation of the procedure involved in the study and provided informed consent. The approval of the implementation of the study was sought from the ethical review committee of Tilganga Institute of Ophthalmology, Kathmandu. The study protocol adhered to the provision of the Declaration of Helsinki for research involving human subjects.

\section{Intervention}

All patients' eyes were cleaned with sterile cotton and ofloxacin $(0.3 \%)$ eye drops were applied 10 minutes before the procedure. Patients were laid supine on the operating table and proparacaine $0.5 \%$ eye drops was applied for topical anesthesia. Visual axis was documented in both eyes. Docking or ocular alignment was done as per the visual axis and suction was applied. Carl Zeiss VisuMax femtosecond laser (Carl Zeiss Meditec, Jena, Germany) was applied for lenticule or flap creation and for superficial corneal incision. Both nasal and temporal $2 \mathrm{~mm}$ incisions were made superiorly. Using the dissector, the lenticule was separated and taken out with forceps in the SMILE procedure, while the flap was separated and lifted, exposing the anterior stroma for ablation in LASIK. In LASIK, Carl Zeiss MEL 80 was used for photoablation and the flap was replaced back. A slit-lamp examination was performed in the operating room to check for any interface particles and the wound site. Postoperatively, patients were observed for an hour and discharged with proper medication instructions (prednisolone eye drops $1 \%$ four times a day, tobramycin eye drops $0.3 \%$ four times a day, ofloxacin eye drops $0.3 \%$ four times a day, sodium carboxymethylcellulose $0.5 \%$ every hour).

\section{Assessment}

Assessment included measurement of visual acuity in internally illuminated Bailey Lovie logMAR chart at $4 \mathrm{~m}$ distance, objective refractive status with Topcon Auto Kerato-Refractometer (KR-8900), subjective refraction to find out subjective acceptance of refractive error, best spectacle corrected visual acuity before surgery, and uncorrected visual acuity after surgery. Subjects' preoperative variables (corneal power, corneal astigmatism, and corneal thickness) were 
obtained through corneal topography (Atlas 9000) and Oculus Pentacam 70700 (SN 3951 5001). Corneal thickness was measured with OptoVue pachymetry (RTVue-100 Fourier domain OCT) version 6.9.

Contrast sensitivity function was measured with functional vision analyzer (Vision Tester 6500P; Stereo Optical Company Inc., Chicago, IL). In this test, the grating patches on rows A to E correspond to spatial frequencies of 1.5, 3, 6, 12 , and 18 cycles/deg. The patient scanned the patches from left to right and reported the last pattern recognized in each row and this response was recorded. The testing instrument was fixed at photopic condition $\left(85 \mathrm{~cd} / \mathrm{m}^{2}\right)$. The contrast sensitivity function was measured at various spatial frequencies presurgery with best optical correction and postsurgery unaided at 3 and 12 months. The average functional acuity contrast test scores for each spatial frequency were recorded and the contrast sensitivity function graph was plotted.

All data were evaluated using Statistical Package for the Social Sciences (version 16.0). Differences between preoperative and postoperative contrast sensitivity at each spatial frequency were analyzed through parametric paired Student's t-test. The $p$-value was considered significant at 0.05 for $95 \%$ confidence interval.

\section{Results}

The mean age of the subjects undergoing refractive surgery was $23.7 \pm 4.4$ years (range: 18-32 years) including 11 males and 4 females. Distribution of preoperative and postoperative refractive error is given in Table 1. The mean spherical equivalent myopia (MSEM) was $-4.68 \pm 1.74 \mathrm{DS}$ in the right eye, $-4.88 \pm 1.71 \mathrm{DS}$ in the left eye, and $-4.78 \pm 1.72$ DS in both eyes preoperatively. MSEM was $-3.2 \pm$ 0.8 (range -2.00 DS to $-4.50 \mathrm{DS}$ ) for FS-LASIK and $-5.9 \pm 1.2$ (range -4.00 DS to $-7.50 \mathrm{DS}$ ) for SMILE. Postoperative residual refractive error that was recorded in 3 and 12 months was clinically insignificant and slightly at the hypermetropic side. All of the patients after refractive surgery had refractive error less than +0.75 DS of hyperopia postoperatively. Emmetropia was noted in 4 eyes (13.33\%), whereas residual refractive error of $+0.12 \mathrm{DS},+0.25 \mathrm{DS},+0.50 \mathrm{DS}$, and $+0.75 \mathrm{DS}$ was noted in $13(43.33 \%), 4(13.33 \%), 8(26.66 \%)$, and 1 (3.33\%) eyes, respectively, postoperatively. The postoperative refractive error was stable over the period of 12 months. Preoperative best spectacle corrected visual acuity was in the range of 20/20 to $20 / 16$ in all the subjects. Postoperative visual acuity was 20/20 or better in 17 subjects (56.7\%), 20/20 in 12 subjects (40.0\%), and 20/25 in 1 subject (3.3\%).

The mean corneal thickness of 30 eyes so included in the study was $528.07 \pm$ 18.49 microns, which after corneal refractive surgery became $444.43 \pm 23.34$ microns. The average change in corneal thickness after LASIK was $69.58 \pm 16.23$ microns, whereas after SMILE it was $93 \pm 25.32$ microns. 
Pradhan, Pradhan, Shrestha, Dhunganga, Kaiti

Table 1. Distribution of mean spherical equivalent refractive error

\begin{tabular}{|l|l|l|l|}
\hline \multirow{2}{*}{ Characteristics } & \multicolumn{3}{|l|}{ Spherical equivalent refractive error } \\
\cline { 2 - 4 } & Right eye & Left eye & Both eyes \\
\hline Preoperative & $-4.68 \pm 1.74$ DS & $4.88 \pm 1.71$ DS & $4.78 \pm 1.72$ DS \\
\hline 3 months postoperative & $+0.29 \pm 0.20$ DS & $+0.27 \pm 0.23$ DS & $+0.28 \pm 0.21$ DS \\
\hline 12 months postoperative & $+0.20 \pm 0.19$ DS & $+0.21 \pm 0.17$ DS & $+0.21 \pm 0.18$ DS \\
\hline
\end{tabular}

\section{Contrast sensitivity function after refractive surgeries}

Figure 1 represents contrast sensitivity functions after refractive surgeries. Overall, contrast sensitivity function was found to be equally improved in 3 months and 1 year after the surgeries at spatial frequencies of 3,12, and 18 cycles per minute (Fig. $1 \mathrm{~A}$ and Table 2). Contrast sensitivity after LASIK surgery was noted to be equally improved in 3 months and 1 year at spatial frequencies of 12 and 18 cycles per minute. Though the graph sloped down in 1 year follow-up at spatial frequency of 6 cycles/minute, the contrast sensitivity function was insignificantly better than preoperative values (Fig. 1B and Table 2). Though contrast sensitivity function was improved after SMILE, this improvement was statistically insignificant at all the spatial frequencies (Fig. 1C and Table 2).

\section{Discussion}

The present study showed that both SMILE and FS-LASIK were predictable for the correction of myopia and myopic astigmatism and had better outcome of visual acuity. Myopic regression was seen in none of the cases included in this study for 12 months.
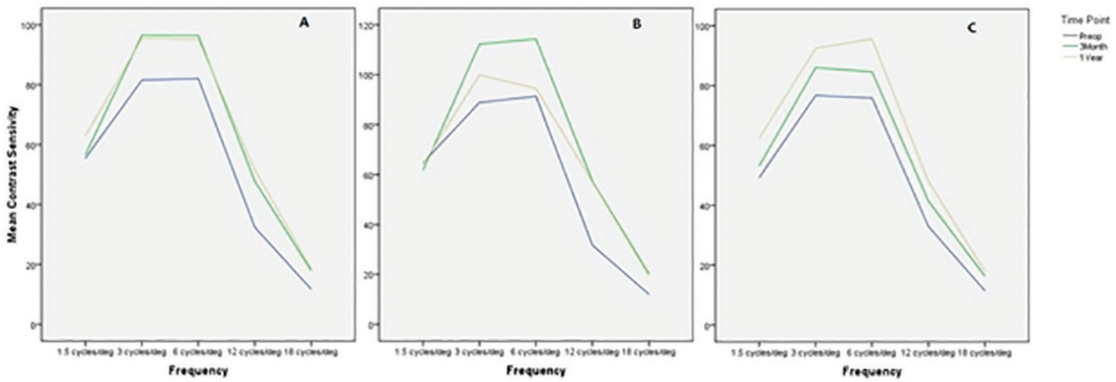

Fig. 1. Preoperative and postoperative contrast sensitivity in FS-LASIK and SMILE.

(A: Overall; B: FS-LASIK; C: SMILE). 
Table 2. Difference between preoperative and postoperative contrast sensitivity function at each spatial frequency

\begin{tabular}{|l|l|l|l|l|l|l|}
\hline \multirow{2}{*}{$\begin{array}{l}\text { Spatial frequency } \\
\text { (cycles/min) }\end{array}$} & \multicolumn{3}{|l|}{ Overall } & \multicolumn{2}{l|}{ FS-LASIK } & \multicolumn{2}{l|}{ SMILE } \\
\cline { 2 - 7 } & $\mathbf{3}$ months & 1 year & 3 months & 1 year & 3 months & 1 year \\
\hline 1.5 & 0.1816 & 0.8149 & 0.7249 & 0.9435 & 0.5474 & 0.0987 \\
\hline 3.0 & $0.0149^{\mathrm{a}}$ & $0.0264^{\mathrm{a}}$ & 0.0572 & 0.2404 & 0.1478 & 0.06779 \\
\hline 6.0 & 0.0853 & 0.1868 & 0.1114 & 0.8113 & 0.4073 & 0.1684 \\
\hline 12.0 & $0.0113^{\mathrm{a}}$ & $0.0021^{\mathrm{a}}$ & $0.0309^{\mathrm{a}}$ & $0.0149^{\mathrm{a}}$ & 0.1880 & 0.0602 \\
\hline 18.0 & $0.0494^{\mathrm{a}}$ & $0.0056^{\mathrm{a}}$ & $0.0450^{\mathrm{a}}$ & $0.0175^{\mathrm{a}}$ & 0.2924 & 0.0746 \\
\hline
\end{tabular}

aSignificantly different at $\mathrm{p} \leq 0.05$ by a paired t-test

There was statistically insignificant increment in contrast sensitivity function after refractive surgery in our study. Preoperative contrast sensitivity was assessed in all subjects with refractive correction in trial lenses. Montés-Micó et al. reported no significant change in the level of contrast sensitivity under photopic conditions and dramatic reduction in contrast sensitivity under mesopic conditions. ${ }^{25}$ We assessed contrast sensitivity in photopic conditions only. Under photopic conditions, contrast sensitivity of postoperative FS-LASIK patients did not differ from normal. In fact, the study showed improved contrast sensitivity function at high spatial frequencies (12 and 18 cycles/deg) after FS-LASIK. This outcome concurs with that reported by Pérez-Santonja and colleagues ${ }^{26}$ and Montés-Micó and Charman, who found no statistically significant decrease in photopic contrast sensitivity at any spatial frequency 3 and 6 months after FS-LASIK. ${ }^{24}$

During early refractive surgery techniques and laser technologies, the quality of vision as measured by contrast sensitivity was typically lower after surgery. The introduction of customized wavefront LASIK greatly improved the accuracy of the laser surgery techniques, resulting in tremendous improvements in the quality of vision. ${ }^{27}$ The Tuan et al. study showed an improvement in contrast sensitivity in a significant percentage of patients following custom LASIK. ${ }^{28}$

Similarly, contrast sensitivity function did not differ postoperatively after SMILE surgery under photopic conditions in our study. Prior studies also suggest that visual function does not alter even after laser refractive correction when evaluated at photopic state. ${ }^{25}$

While comparing the contrast sensitivity between FS-LASIK and SMILE, there was not much of a difference at low spatial frequencies prior to the surgery. However, at high spatial frequencies, contrast function seemed to improve 
significantly after FS-LASIK compared to SMILE. The probable reason could be a smoother interface achieved after photoablation of the tissue with excimer laser in FS-LASIK.

This study only compared contrast sensitivity under photopic conditions among the subjects undergoing FS-LASIK and SMILE. So the application of the study is limited as no mesopic and scotopic conditions were maintained and compared. Generalization is also nominal, the sample size being very small. Important information such as topographic measurement data and tear function tests is missing in the study as it is being a retrospective study. Further prospective study is warranted considering all these variables and measuring them especially under mesopic and scotopic conditions.

Contrast sensitivity function is an important tool to measure visual function, which may be altered after multitude of refractive surgeries. Under photopic conditions, the contrast sensitivity function remains the same or becomes slightly better after refractive surgery.

\section{References}

1. Lohmann CP, Garty DS, Muir MK, Timberlake GT, Fitzke FW, Marshall J. Corneal haze after excimer laser refractive surgery: objective measurements and functional implications. Eur J Ophthalmol. 1991;1:173-180.

2. Ficker $L A$, Bates $A K$, Steele $A D$, et al. Excimer laser photorefractive keratectomy for myopia: 12 months follow-up. Eye. 1993;7:617-624.

3. Dutt S, Steinert RF, Raizman MB, Puliafito CA. One-year results of excimer laser photorefractive keratectomy for low to moderate myopia. Arch Ophthalmol. 1994;112:1427-1436.

4. Ambrosio G, Cennamo G, De Marco R, Loffredo L, Rosa N, Sebastiani A. Visual function before and after photorefractive keratectomy for myopia. Refract Corneal Surg. 1994;10:129-136.

5. Butuner Z, Elliott DB, Gimbel HV, Slimmon S. Visual function one year after excimer laser photorefractive keratectomy. Refract Corneal Surg. 1994;10:625-630.

6. Halliday BL. Refractive and visual results and patients satisfaction after excimer laser photorefractive keratectomy for myopia. Br J Ophthalmol. 1995;79:881-887.

7. Lafond G. Treatment of halos after photorefractive keratectomy. J Refract Surg. 1997;13:83-88.

8. Ghaith AA, Danile J, Stulting RD, Thompson KP, Lynn M. Contrast sensitivity and glare disability after radial keratotomy and photorefractive keratectomy. Arch Ophthalmol. 1998;116:12-18.

9. Holladay JT, Dudeja DR, Chang J. Functional vision and corneal changes after laser in situ keratomileusis determined by contrast sensitivity, glare testing, and corneal topography. J Cataract Refract Surg. 1999;25:663-669.

10. Nordan LT, Slade SG, Baker RN, Suarez C, Juhasz T, Kurtz R. Femtosecond laser flap creation for laser in situ keratomileusis: six-month follow-up of initial U.S. clinical series. J Refract Surg. 2003;19:8-14.

11. Kim JY, Kim MJ, Kim TI, Choi HJ, Pak JH, Tchah H. A femtosecond laser creates a stronger flap than a mechanical microkeratome. Invest Ophthalmol Vis Sci. 2006;47:599-604.

12. Binder PS. One thousand consecutive IntraLase laser in situ keratomileusis flaps. J Cataract Refract Surg. 2006;32:962-969.

13. Montes-Micó R, Rodríguez-Galietero A, Ali ó JL. Femtosecond laser versus mechanical keratome LASIK for myopia. Ophthalmology. 2007;114:62-68.

14. Sugar A. Ultrafast (femtosecond) laser refractive surgery. Curr Opin Ophthalmol. 2002;13:246-249. 
15. Krueger RR, Trokel SL, Schubert HD. Interaction of ultraviolet laser light with the cornea. Invest Ophthalmol Vis Sci. 1985;26:1455-1464.

16. Pellin MJ, Williams GA, Young CE, Gruen DM, Peters MA. Endoexcimer laser intraocular ablative photodecomposition. Am J Ophthalmol. 1985;99:483-484.

17. Marshall J, Sliney DH. Endoexcimer laser intraocular ablative photodecomposition. Am J Ophthalmol. 1986;101:130-131.

18. McDonald MB, Deiz MR, Frantz JM, et al. Photorefractive keratectomy for low-to-moderate myopia and astigmatism with a small-beam, tracker-directed excimer laser. Ophthalmology. 1999;106:1481-1488.

19. Shah R, Shah S. Effect of scanning patterns on the results of femtosecond laser lenticule extraction refractive surgery. J Cataract Refract Surg. 2011;37:1636-1647.

20. Reinstein DZ, Archer TJ, Gobbe M. Small incision lenticule extraction (SMILE) history, fundamentals of a new refractive surgery technique and clinical outcomes. Eye Vision. 2014;1:3.

21. Van den Berg TJ. Importance of pathological intraocular light scatter for visual disability. Doc Ophthalmol. 1986;61:327-333.

22. Wachler BS, Durrie DS, Assil KK, Krueger RR. Role of clearance and treatment zones in contrast sensitivity: significance in refractive surgery. J Cataract Refract Surg. 1999;25:16-23.

23. Montés-Micó R, Charman WN. Mesopic contrast sensitivity function after excimer laser photorefractive keratectomy. J Refract Surg. 2002;18:9-13.

24. Montés-Micó R, Charman WN. Choice of spatial frequency for contrast sensitivity evaluation after corneal refractive surgery. J Refract Surg. 2001;17:646-651.

25. Montés-Micó R, España E, Menezo JL. Mesopic contrast sensitivity function after laser in situ keratomileusis. J Refract Surg. 2003;19:353-356.

26. Pérez-Santonja JJ, Sakla HF, Alió JL. Contrast sensitivity after laser in situ keratomileusis. J Cataract Refract Surg. 1998;24:183-189.

27. Huang SCM, Chen HCJ. Overview of laser refractive surgery. Chang Gung Med J. 2008;31:237-252.

28. Tuan KM, Liang J. Improved contrast sensitivity and visual acuity after wavefront-guided laser in situ keratomileusis: in-depth statistical analysis. J Cataract Refract Surg. 2006;32(2):215-20. 